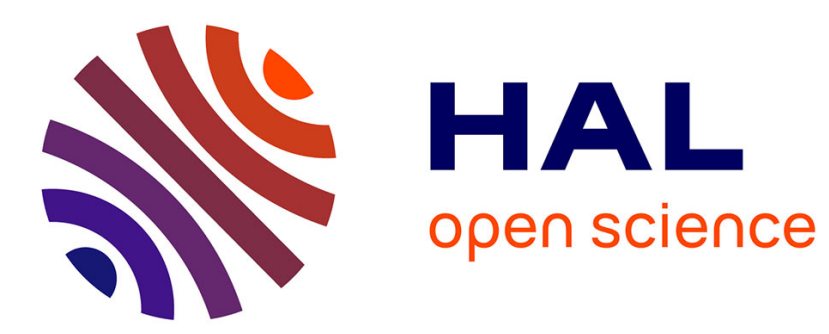

\title{
SAXS study of nucleation and growth of CdTe1-xSx semiconductor nanocrystals in borosilicate glass
}

\author{
A. Craievich, O. Alves, L. Barbosa
}

\section{To cite this version:}

A. Craievich, O. Alves, L. Barbosa. SAXS study of nucleation and growth of CdTe1-xSx semiconductor nanocrystals in borosilicate glass. Journal de Physique IV Proceedings, 1993, 03 (C8), pp.C8-373-C8376. 10.1051/jp4:1993877. jpa-00252308

\section{HAL Id: jpa-00252308 https://hal.science/jpa-00252308}

Submitted on 1 Jan 1993

HAL is a multi-disciplinary open access archive for the deposit and dissemination of scientific research documents, whether they are published or not. The documents may come from teaching and research institutions in France or abroad, or from public or private research centers.
L'archive ouverte pluridisciplinaire HAL, est destinée au dépôt et à la diffusion de documents scientifiques de niveau recherche, publiés ou non, émanant des établissements d'enseignement et de recherche français ou étrangers, des laboratoires publics ou privés. 


\title{
SAXS study of nucleation and growth of $\mathrm{CdTe}_{1_{-x}} \mathrm{~S}_{x}$ semiconductor nanocrystals in borosilicate glass
}

\author{
A.F. CRAIEVICH, O.L. ALVES ${ }^{*}$ and L.C. BARBOSA** \\ Laboratório Nacional de Luz Síncrotron/CNPq, Cx. Postal 6192, 13081-970 Campinas, SP, Brasil \\ * Instituto de Química/UNICAMP, Cx. Postal 6154, 13081-970 Campinas, SP, Brasil \\ ** Instituto de Física/UNICAMP, CX. Postal 6165, 13081-970 Campinas, SP, Brasil
}

\begin{abstract}
Previous investigations demonstrated that borosilicate glasses containing CdTeS nanocrystals exhibit quantum confinement effects. In this paper nucleation and growth of $\mathrm{CdTe}_{1-x} \mathrm{~S}_{\mathrm{x}}(\mathrm{x}=0,0.40$ and 0.70$)$ spheroidal nanocrystals (with radii of a few nanometers) were studied during isothermal annealing at $580 \mathrm{C}$ using the SAXS technique. The time evolutions of the averages of nanocrystal radii and width of the size distribution depend on the crystal stoichiometry. Smaller crystals are formed when sulfur content is lower. The rate of growth increases with sulfur content. The geometrical standard deviation of the radii of ternary crystals exhibits a minimum at annealing times of 15-20 min. These results provide useful hints which can help to obtain glass-CdTeS nanocrystal composites having a controlled average crystal size and a narrow size distribution.
\end{abstract}

1. Introduction - A number of glasses doped with semiconductor nanocrystals with characteristic sizes of a few nanometers exhibit non-linear optical properties. Several glasses containing semiconductor crystallites of $\mathrm{CdSe}_{(1-x)} S_{x}$, of a few nanometers in diameter, have been studied extensively by many authors $(1,2)$. This work deals with a more recently obtained material based on a borosilicate host glass matrix containing $\mathrm{CdTe}_{(1-x)} \mathrm{S}_{x}$ nanocrystals. In order for this type of material to exhibit the so-called quantum confinement effect, the semiconductor nanocrystals should have a size smaller than the electronic Bohr radius. This radius is equal to 5.5 $\mathrm{nm}$. for $\mathrm{CdSe}_{(1-x)} \mathrm{S}_{\mathrm{x}}$ and to $7.4 \mathrm{~nm}$. for $\mathrm{CdTe}_{(1-x)} \mathrm{S}_{\mathrm{x}}$. This suggests that the quantum confinement effect can more easily occur in glasses containing CdTeS crystals(3). Previous transmission electron microscopy and electron diffraction studies on this system (4) demonstrated that the semiconductor particles are actually crystalline and exhibit a spherical shape. These studies also proved that the nanocrystals have a size dispersion and that the size distribution function is a single mode distribution. For many applications, such as low frequency band pass filters in the visible, the variation in optical transmittance of the material should be as abrupt as possible. This is obtained in practice when the size distribution of the nanocrystals is very narrow.

2. Sample preparation - The borosilicate glass-semiconductor composites were obtained by melting the raw materials $\left(\mathrm{SiO}_{2}-\mathrm{H}_{3} \mathrm{BO}_{3}, \mathrm{Na}_{2} \mathrm{CO}_{3}-\mathrm{ZnO}\right.$ with additions of $\mathrm{CdO}, \mathrm{Te}$ and $\mathrm{S}$ to produce the different stoichiometries) in a radiofrequency furnace at $1400 \mathrm{C}$ under a controlled atmosphere. After homogenization, the melts were quenched down to room temperature. The quenched materials were then shaped to obtain thin plates adequate for SAXS measurements and then annealed during different periods of time at $580 \mathrm{C}$.

3. Technique and methods - The SAXS experiments were performed using the D24 synchrotron radiation workstation at LURE, Orsay. This experimental setup is composed of an asymmetrically-cut bent $\mathrm{Si}$ crystal and several slits which provide a monochromatic $X$-ray beam with point-like cross-section and wavelength $\lambda=1.60 \AA$. A position-sensitive X-ray detector was used to record the scattered X-ray photons. The sample-detector distance was $100 \mathrm{~cm}$. A vacuum path between the sample and the detector reduced the parasitic scattering from air. Since the cross-section of the incident beam was very small, the experimental SAXS curves did not need any desmearing mathematical treatment.

A specially designed high temperature sample chamber(5) made possible kinetic in-situ SAXS studies of nucleation and growth of $\mathrm{CdT}_{0.30} \mathrm{~S}_{0.70}$ and $\mathrm{CdTe}_{0.60} \mathrm{~S}_{0.40}$ crystals immersed in a borosilicate glass matrix. The counting times were kept low (typically $200 \mathrm{sec}$.) as compared to the total annealing time (about 1 hour). Other samples containing binary CdTe crystals were studied by SAXS after ex-situ annealing treatments.

The SAXS intensity curves were plotted as functions of the module of the scattering vector $q=4 \pi \sin \theta / \lambda$, where $\theta$ is half of the scattering angle. The parasitic scattering was subtracted from the experimental intensity before further analysis. This subtraction did not add significant errors in most SAXS results, except at very low-q values for which the parasitic scattering exhibits a strong increase, and also at very high q, at which the SAXS intensity from the sample is very weak. 
The basic theory of SAXS applied in this work involves the determination of a set of parameters related to several average values of the radii of the semiconductor crystals. The nanocrystals are supposed to be spherical, to have a single mode distribution in size and to form a "dilute" system. These assumptions are justified by the conclusions of the previous electron microscopy study already mentioned(4). The parameters $R_{g}, R_{v}$ and $R_{p}$, related to three different averages of the nanocrystal radii, were determined from the SAXS intensity $1(q)$ by means of the following equations $(6)$ :

$$
\begin{array}{llll}
R_{g}=(5 / 3)^{1 / 2}<R_{G}>=\left[<R^{8}>/<R^{6}>\right]^{1 / 2} & \text { where } & <R_{G}>=3^{1 / 2}\left\{-\lim \left[d \text { ln } \mid(q) / d\left(q^{2}\right)\right](q \rightarrow 0)\right\}^{1 / 2} & \text { (Guinier law) } \\
R_{V}=(3 / 4 \pi)^{1 / 3}<V>1 / 3=\left[<R^{6}>/<R^{3}>\right]^{1 / 3} & \text { where } & <V>=2 \pi^{2} \mid(0) / Q & \text { and } \left.\quad Q=\int_{0}^{\infty} q^{2} \mid(q) d q\right)
\end{array}
$$

$R_{p}=(3 / 4)<D>=<R^{3}>/<R^{2}>\quad$ where $\quad<D>=(4 / \pi) \int_{0}^{\infty} q^{2} l(q) d q / K$ and $K=l i m\left[l(q) q^{4}(q \rightarrow \infty)\right] \quad$ (Porod law)

The knowledge of the three average radii given in equations $1\left(R_{g}\right.$ : Guinier average), $2\left(R_{v}\right.$ : volume average) and 3 $\left(R_{p}\right.$ : Porod average) permits the determination of other radius averages and a parameter related to the dispersion in size when the radius distribution can be reasonably well approximated by a simple two-parameter function. This was done in our case by assuming, as in Ref. (6), a single mode distribution represented by a log-normal function:

$$
\mathrm{N}(\mathrm{R})=\frac{1}{\mathrm{R}(2 \mu)^{1 / 2} \ell \mathrm{n} \sigma} \exp \left[-\frac{(\ell \mathrm{n} R-\ell \mathrm{n} \mu)^{2}}{2 \ln ^{2} \sigma}\right]
$$

where $\mu$ is the geometric average value of the sphere radius and $\sigma$ is the geometric standard deviation of the radii distribution. These parameters are calculated by plotting $R_{i}\left(R_{p}, R_{v}\right.$ and $\left.R_{g}\right)$ as a function of $k_{i}(i=p, v, g)$ which are equal to 2.5, 4.5 and 7.0 for $\mathrm{k}_{\mathrm{p}}, \mathrm{k}_{\mathrm{v}}$ and $\mathrm{k}_{\mathrm{g}}$, respectively. If the log-normal function well represents the real distribution of crystal sizes, $R_{i}$ will depend linearly on $k_{j}(6)$ :

$$
\ell n \mathrm{R}_{\mathrm{i}}=\ell \mathrm{n} \mu+\ell \mathrm{n}^{2} \sigma \mathrm{k}_{\mathrm{i}}
$$

The parameters $\mu$ and $\sigma$ can be determined by fitting straight lines to $\ell n R_{j}$ vs $k_{i}$ plots.

4. Experimental results - The first in-situ kinetic SAXS study was carried out on thin plates of borosilicate glass$\mathrm{CdTe}_{0.30} \mathrm{~S}_{0.70}$ crystal samples held at $580 \mathrm{C}$ for different annealing times. The resulting $\mathrm{X}$-ray scattering curves are plotted in Fig. 1. The origin of the observed SAXS intensity was attributed to the electron density contrast due to the formation of spheroidal CdTeS nanocrystals in the glass matrix. The scattering intensity and its q-dependence exhibit strong variations for increasing annealing times. SAXS measurements on as-quenched samples of the same composition, at room temperature, exhibited a practically constant and weak SAXS intensity, indicating that the asquenched material is essentially homogeneous and only contains weak and short range electronic density fluctuations. The q-dependence of the SAXS curve, corresponding to an annealing time of $\mathrm{t}=2 \mathrm{~min}$ (Fig. 1), indicates that, even for this short time, the sample evolved significantly and small crystals had already formed.

The variations in SAXS intensity functions in Fig. 1 suggest a rapid growth of the nanocrystals from the very early stages of the process. The maximum in SAXS intensity on the low-q side of the curve corresponds to $t=20$ min. After this period of time the intensity decreases along all the q-range. Ln I vs. $q^{2}$ plots of these curves exhibit a linear domain at low $q$ from which the Guinier average radius and its variation with time were determined.

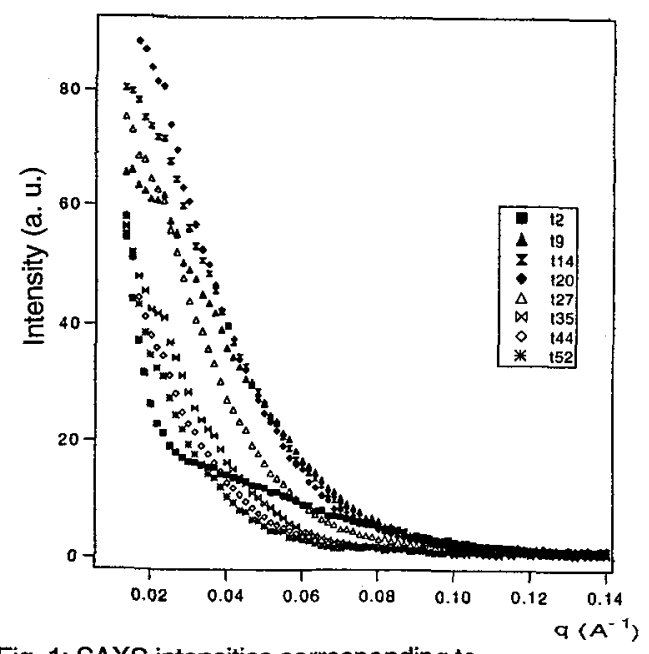

Fig. 1: SAXS intensities corresponding to $\mathrm{CdTe}_{0.30} \mathrm{~S}_{0.70}$ nanocrystals in a borosilicate glass matrix for the indicated annealing time (t $\mathrm{min})$.

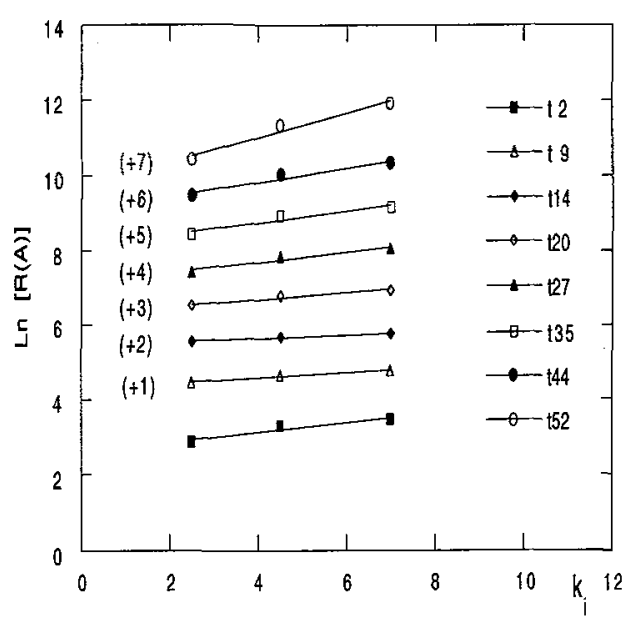

Fig. 2: $L n R_{i}$ vs. $k_{i}$ plots of $C d T e_{0.30} S_{0.70}$ for different annealing time ( $\mathrm{t} \mathrm{min}$ ). The scale for $\ell n \mathrm{R}_{\mathrm{i}}$ applies to the $\mathrm{t} 2$ curve; the others are vertically displaced as indicated. 
The SAXS curve corresponding to 2 min of annealing time (Fig. 1) exhibits a singular behavior characterized by a strong variation in intensity at very small $\mathrm{q}$. This may be associated in this particular case either with a bimodal size distribution due to a transient effect in the crystal nucleation and growth process or to a possible systematic error in parasitic intensity subtraction. Since a model of a single mode size distribution was assumed, the small low-q domain of the SAXS curve for $t=2$ min. was not included in the calculation of $\left\langle R_{G}\right\rangle, 1(0)$ and $Q$ (eq. 1, 2 and 3 ).

Another unexpected result concerns the decrease in SAXS intensity for advanced stages of crystal growth. This is probably due to an increase in sample thickness during annealing related to an incipient glass flow. The existence of this effect impeded us from doing any quantitative comparison between the SAXS intensities produced by the sample at different annealing times. We have determined $R_{g}, R_{v}$ and $R_{p}$ (eqs. 1, 2 and 3 ) because these parameters are not sensitive to variations in sample thickness.

Using Porod's plots of SAXS intensity [I(q) $q^{4}$ vs. $\left.q^{4}\right]$, the parameters $\mathrm{K}$ (eq. 3 ) were determined for every annealing time. A constant intensity value was subtracted from the measured SAXS intensity in order to suppress the small contribution from short range electronic density fluctuations in the matrix.

Plots of $\ell n R_{i}$ vs. $k_{i}$ corresponding to a glass containing CdTe $e_{0.30} S_{0.70}$ nanocrystals are shown in Fig. 2 for different annealing times. From these plots and according to eq. 5, the values of $\mu$ (geometrical average of nanocrystal radii) and $\sigma$ (geometrical standard deviation) were determined for every annealing time.

An equivalent kinetic study was performed on the borosilicate glass-CdTe $0.60 S_{0.40}$ crystal system. The SAXS curves corresponding to advanced stages of crystal formation exhibited, at very low $q$, a strong variation in intensity similar to that observed for the sample with a composition $x=0.70$ and 2 min. of annealing time. Again, because of the assumption of a single mode size distribution, the low-q domain of these curves was not included in the data analysis. From the several plots of $\ell n R_{i} v s . k_{i}$, the functions $\mu(t)$ and $\sigma(t)$ were determined.

A borosilicate glass containing CdTe (binary) crystals was studied by SAXS under as-quenched condition and after ex-situ annealing at $580 \mathrm{C}$ during 1 hour. Since the scattering intensity from this sample is very weak, the only parameter determined from the experimental results was the Guinier average radius (eq.1).

5. Discussion - The time variations of Guinier average radius of nanocrystals, $R_{\mathrm{g}}$, are plotted in Fig. $3 a$ for the three studied crystal compositions. A monotonic increase in the Guinier average radii corresponding to the two ternary stoichiometries is apparent during the first stages of crystal growth. The Guinier average radius of the binary CdTe nanocrystals is much smaller than those of ternary crystals for equivalent annealing time. At early stages of crystal formation, the growth is faster for higher sulfur content. In samples with higher sulfur content $(X=0.70)$, the Guinier average radius increases even for well-advanced annealing stages. In the case of crystals with $x=0.40, R_{g}(t)$ reaches an upper limit at $\mathrm{t}=20 \mathrm{~min}$. and then remains constant.

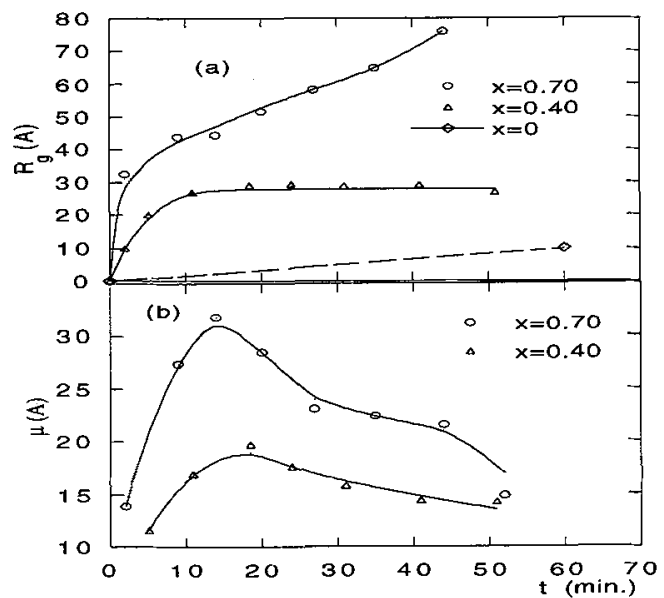

Fig. 3: Time evolutions of Guinier average radius (a) and geometric average radius (b) of nanocrystals.

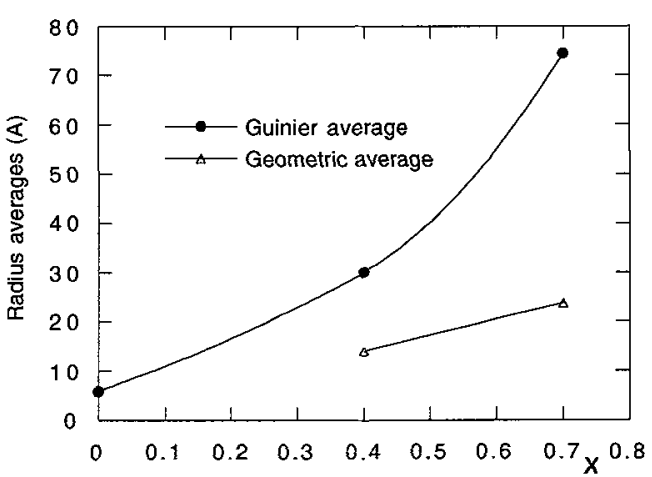

Fig. 4: Guinier averages, $R_{g}$, and geometric averages, $\mu$, of nanocrystal radii for different crystal composition corresponding to an equivalent annealing time ( $\mathrm{t}=45 \mathrm{~min}$.).

The geometric averages $\mu$ of crystal radii were plotted as functions of time in Fig. $3 b$ for $C d T e_{0.30} S_{0.70}$ and $\mathrm{CdTe}_{0.60} \mathrm{~S}_{0.40}$ crystal stoichiometries. The parameter $\mu$ is higher for $\mathrm{x}=0.70$ than $\mathrm{x}=0.40$ along all annealing times. The function $\mu(t)$ exhibits a maximum which was not apparent in the plot of $R_{g}(t)$. The behaviors of $\mu(t)$ and $R_{g}(t)$ are not similar because they are very different averages of a varying distribution function. Guinier average radius weighs the large crystals much more strongly than the geometric average.

The Guinier average radii, $\mathrm{R}_{\mathrm{g}}$, and the geometric averages, $\mu$, corresponding to different sample stoichiometries and equivalent annealing time ( $t=45 \mathrm{~min}$.), are plotted in Fig. 4. The significant differences between $R_{g}$ and $\mu$ for $x=0.40$ and $x=0.70$ indicate a rather wide distribution in crystal size. Both crystal radius averages $\left(R_{g}\right.$ and $\mu)$ are higher in samples with higher sulfur content along all the crystal growth process. 
The geometrical standard deviations, $\sigma$, of the nanocrystal radius distributions are plotted in Fig. 5 for the two ternary crystals. We infer from these plots that $\sigma$ initially decreases down to a minimum and then increases monotonically for both compositions. The minimum in $\sigma$ occurs between 15 and 20 min for both compositions.

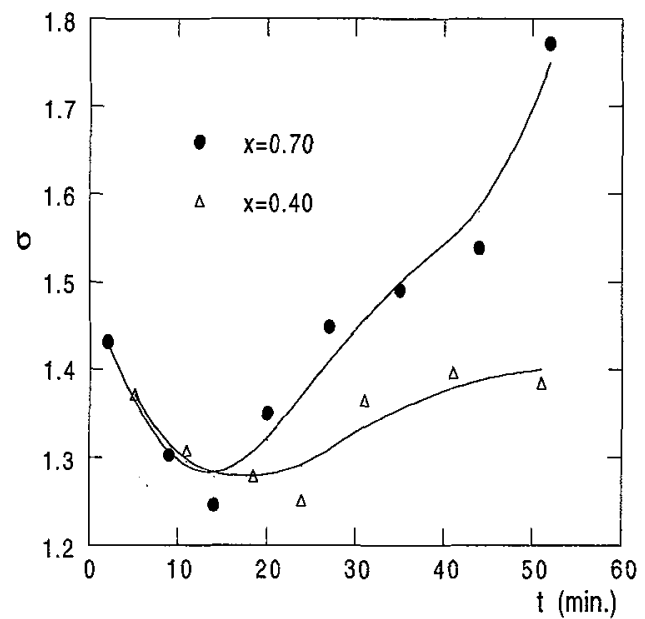

Fig. 5: Geometric standard deviations of crystal radii corresponding to samples with the indicated sulfur content ( $x)$.

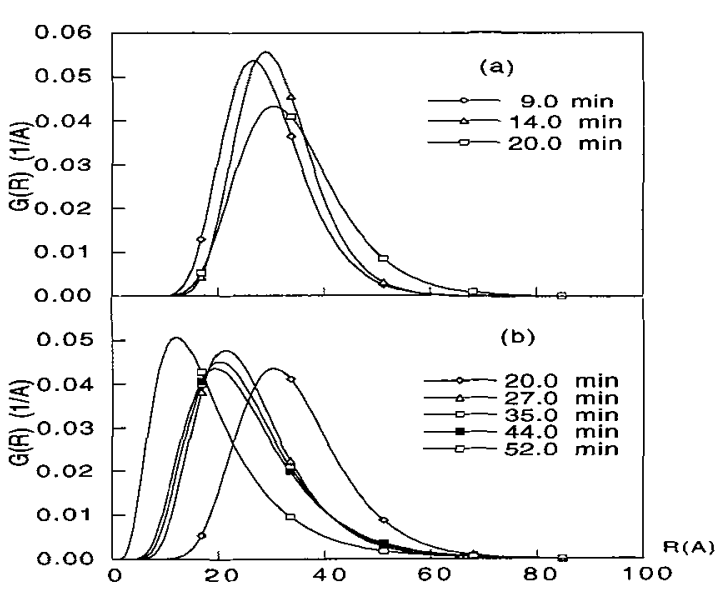

Fig. 6: Normalized radius distribution functions of the $\mathrm{CdTe}_{0.30} \mathrm{~S}_{0.70}$ nanocrystals corresponding to early (a) and advanced (b) stages of crystal growth.

The $G(R)$ functions plotted in Fig. $6 a$ and $6 \mathrm{~b}$ correspond to ternary crystals with $x=0.70$. They were calculated using in eq. 4 the values of the parameters $\mu$ and $\sigma$ given in Fig. 3 and 5 , respectively. The size distribution functions related to initial and advanced stages of crystal growth are plotted separately (Fig. 6a and 6b, respectively). The $G(R)$ functions corresponding to early stages of crystal growth (Fig. 6a) exhibit a continuous shift toward high $\mathrm{R}$ values. This is qualitatively expected for classic processes of crystal nucleation and growth. Fig. $6 \mathrm{~b}$, corresponding to samples with $\mathrm{x}=0.70$ at advanced stages of heat treatment, indicates a progressive shift of the $G(R)$ functions towards small $R$ values. At advanced stages, the classic mechanism of coarsening (the bigger crystals growing at the expense of smaller ones) is expected to be predominant. Since coarsening produces a continuous growth of the large particles and the correlated elimination of the small ones, the size distribution is expected to shift towards high $R$ values. The results shown in Fig. $6 \mathrm{~b}$ indicate an opposite behavior and lead us to conclude that coarsening cannot be the only active mechanism during the advanced stages of crystal growth. An equivalent conclusion was inferred from the similar SAXS study of glass samples containing $\mathrm{CdTe}_{0.60} \mathrm{~S}_{0.40}$ nanocrystals.

The results corresponding to advanced stages can be qualitatively understood by assuming that, at the same time that crystal growth occurs, small new crystals are still nucleating. This actually may happen when the annealing temperature is chosen in such a way that the rates of nucleation and growth are both important. During the first stages of the process the rate of crystal growth is rather fast and, even if new crystals still nucleate, the overall size distribution would shift towards high- $\mathrm{A}$ values as shown in Fig. $3 \mathrm{~b}$ and $6 \mathrm{a}$. During the advanced stages, the rate of crystal growth is slow and nucleation of additional small crystals would then produce a decrease in the geometric average size, as shown in Fig. $3 \mathrm{~b}$ and $6 \mathrm{~b}$. These statements must be considered as a tentative and qualitative explanation of the presented experimental results. In order to confirm them, additional studies are needed.

6. Conclusion - The main conclusions of this study on the kinetics of CdTeS nanocrystal growth in borosilicate glass during isothermal annealing at $580 \mathrm{C}$ are: $i)$ the Guinier, $\mathrm{R}_{\mathrm{g}}$, and the geometric, $\mu$, averages of nanocrystal radii increase with increasing sulfur content, ii) the behavior of the $\mu(t)$ function is characterized by an initial increase and further decrease, which seem to be due to the simultaneous occurrence of crystal nucleation and crystal growth even at advanced stages of annealing, and iii) the geometrical standard deviation of crystal radii, $\sigma$, exhibits a minimum after a transient decrease, suggesting that an annealing time of 15-20 min is the optimum to minimize this parameter. In order to produce glass-nanocrystal composites exhibiting a narrower crystal size dispersion, a two-step process is suggested: first, at a temperature lower than that of the present investigation, in order to enhance crystal nucleation and reduce the rate of crystal growth, and second, at a higher temperature, to increase the rate of growth of the existing nuclei and suppress or reduce simultaneous crystal nucleation.

(1) EFROS, AL. E and EFROS, A. E, J. Opt. Soc. Am. B2 (1985) 1155.

(2) EKIMOV, A. I. and EFROS, A. E., Soc. Phys. Semicond. 16 (1982) 772.

(3) RICARD, D., ROUSSIGNOL, P., HACHE, F. and FLYTZANIS, C., Phys. Stat. Solidi B $159(1990) 275$.

(4) MEDEIROS NETO, J. A., BARBOSA, L. C., CESAR, C. L., ALVES, O. L and GALEMBECK, F., Appl. Phys. Lett. 59 (1991) 2715 .

(5) BAGNATO, O. and AUGUSTO, N. V., LNLS Technical Manual 03/92.

(6) WALTER, G., KRANOLD, R., GERBER, T., BALDRIAN, J. and STEINHART, M., J. Appl. Cryst. $18(1985) 205$. 